\title{
Change detection in multitemporal images through single- and multi-scale approaches
}

\author{
Bruno Aiazzi, Francesca Bovolo, Lorenzo Bruzzone, Andrea Garzelli, \\ Davide Pirrone, and Claudia Zoppetti
}

\begin{abstract}
This chapter presents an analysis of the current status and of the challenges in change detection techniques for the analysis of multitemporal SAR images. After a brief review of the recent literature on general change detection methods, the chapter investigates the specific problem of change detection in SAR images. The main properties of the change detection problem in SAR images are explored and discussed. Then, recent change detection techniques for high resolution (HR) and very high resolution (VHR) SAR data are presented and critically analysed from the theoretical viewpoint. Finally, examples of application of these techniques to real problems are presented by using simulated image pairs and Enhanced Spotlight COSMO-SkyMed images.
\end{abstract}

\section{Introduction}

Change Detection $(\mathrm{CD})$ in remote sensing is defined as the process of identifying changes in the features of the scene by means of the joint analysis of pair of images acquired at different times over the same geographical area. CD has several applica-

B. Aiazzi

Institute of Applied Physics "Nello Carrara", National Research Council, Via Madonna del Piano 10, 50019 Sesto Fiorentino, Firenze, Italy e-mail: b.aiazzi@ifac.cnr.it

F. Bovolo and D. Pirrone

Fondazione Bruno Kessler, via Sommarive 18, 38123, Povo, Trento, Italy e-mail: \{bovolo\}\{pirrone\}@fbk.eu

L. Bruzzone

Department of Information Engineering and Computer Science, University of Trento, via Sommarive 9, 38123, Povo, Trento, Italy e-mail: lorenzo.bruzzone@unitn.it

A. Garzelli and C. Zoppetti

Department of Information Engineering and Mathematics, University of Siena, via Roma 56, 53100, Siena, Italy e-mail: $\{$ andrea.garzelli $\}\{$ claudia.zoppetti $\} @$ unisi.it 
tions in environmental monitoring, such as damage assessment or urban expansion monitoring.

Applications of change detection form spaceborne platforms progressively migrate from slowly changing phenomena (land-cover dynamic analysis, deforestation control) to rapidly mapping observations of natural or anthropic disasters, like landslides, floods, earthquake damages, fires, oil pollution, etc. Polar orbiting satellites, however, do not provide adequate revisit time to monitor unpredictable and exceptional events. Therefore, making a direct comparison between post-event and pre-event data having identical acquisition parameters is almost unfeasible [29]. Hence, change detection is performed by comparing the first available data acquired after the event and previously archived acquisitions of the same scene.

One of the main challenges for a change detection algorithm is that the changes produced by the event under observation cannot be easily modeled. Actually, the same kind of event exhibits different signatures, depending on the region where it occurred, e.g., an urban or an agricultural area, and on the characteristics of the imaging sensor. Furthermore, when the time interval between two observations is large, changes to be identified are often mixed to seasonal or incidental changes that may be the majority, even if they usually have a minor extent and are often less relevant from the application viewpoint.

Several different approaches to change detection have been proposed in the literature [4], [10]-[15],[17],[19]-[22], [18, 16][24]-[27],[26, 28, 30], [34]-[36], [43]-[46], [48]-[50], [54]-[56],[58]-[60], [63],[65]-[70].

Several examples can be found of (semi)supervised $[20,22,18,49,65,66$, 70], and unsupervised [4], [10]-[17],[19],[21] methods as well. Labeled samples for each or some of the considered multitemporal acquisitions are required when supervised or semi/partially supervised methods are considered, whereas they are not for unsupervised approaches. Thus the possibility to gather reference samples for the training phase is an element that drives the kind of method to employ. Since the training sample collection is complex or even unfeasible, unsupervised approaches are often preferred. On the other side application requirements should be considered as well. In fact, unsupervised methods do not provide a "from-to" information about the kind of change. Further, unsupervised methods are specifically designed to handle multitemporal images acquired from either active SAR sensors [4, 11, 14],[25]-[27],[30, 34, 36, 43, 46, 48, 56, 58, 63], or optical passive ones $[12,10,13,19,17,21,16,35,44,50,54,55,59,60]$.

Other methods are more general and are able to handle multisensor information $[15,20,22,18,49,50],[65]-[70]$. However, due to the scarce sensitivity of SAR to atmospheric and weather conditions, the available post-event data are likely to be SAR images [37]. Furthermore, the potentials of SAR sensors in change detection applications are strengthened by the high spatial resolution and the short revisit time provided by the new generation SAR-based missions, such as COSMO-SkyMed (CSK), TerraSAR-X (TSX), RadarSat 3 and Sentinel-1. The improvement in spatial resolution, which can reach $1 \mathrm{~m}$ for Spotlight products, is of fundamental importance in case of urban or suburban scenes [57]. In addition, the four-satellite constellation of the CSK system increases the possibility of monitoring the temporal evolution of 
an environmental disaster effectively. A worst-case minimum revisit time of $12 \mathrm{~h}$ is guaranteed.

This chapter starts with an analysis of state of the art that considers change detection methods for both optical passive and SAR active images. After that, attention is devoted to recent change detection techniques for high resolution (HR) and very high resolution (VHR) SAR, with specific attention to the trade-off between effective speckle reduction from SAR data (see also Chapters 4 and 5) and good preservation of the fine spatial details provided by the new generation of spaceborne SAR missions. Both simulated data and COSMO-SkyMed image pairs are considered for experimental evaluation and performance comparison among single-scale and multiscale approaches.

\section{State of the Art}

\subsection{Change Detection in Multitemporal Spaceborne Images}

As mentioned in the introduction, the literature is plenty of methods for change detection both for optical passive and active SAR images. At a given level of abstraction, most of them follow similar philosophies. However, they strongly differ in the implementation details. This is because the statistical model of the two kind of data is different: optical passive image processing relies on an additive Gaussian noise model, whereas SAR image processing relies on a multiplicative speckle noise model.

Among supervised methods three macro groups can be identified: Post Classification Comparison [66], Supervised Direct Multidate Classification [49, 66], and Compound Classification [20, 22, 23, 65, 70]. Post Classification comparison (PCC) (also referred to as delta classification [66]) performs change detection by comparing the classification maps obtained by classifying two images independently. Multitemporal images are independently classified, thereby minimizing the problem of radiometric calibration, but ground truth is required for each of them. Although PCC has been used in several applications extensively, its performance strongly depends on the classification accuracy of the classifiers applied to each single image. Supervised Direct Multidate Classification (DMC) $[49,66]$ characterises pixels by stacking the feature vectors related to the images acquired at the two different times. Each class transition is considered as a single class thus the training pixels should represent the proportions of all the transitions in the whole area of interest accurately. This represents a serious drawback as, in real applications, it is difficult to obtain training sets with such characteristic. A more realistic approach is Compound Classification (CC) [22], since it allows the temporal correlation between images to be considered in the change-detection process. When ground truth is not available for each multitemporal acquisition partially-supervised classifiers can be used. They are able to update the classifier parameters estimated according to the ground truth 
available for one multitemporal image and match them to the statistical properties of multitemporal images for which the ground truth is not available. These methods, recently referred to as Domain Adaptation (DA) methods [18, 24, 31], have been investigated with novel interest because of the use with active learning (AL) [38]-[40]. All the aforementioned methods are based on classifiers like for example Maximum Likelihood classifier [42, 62], Neural Networks [23, 51], Fuzzy Classifiers [9, 39], and Support Vector Machines [71, 33], which are either the most widely used or the most effective ones (the reader is referred to the literature for more details on the behaviour and mathematical details of each single classifier. An example of multitemporal classification of optical images is shown in Chapter 7.). Because of this, such approaches are intrinsically suitable to process data acquired from either passive optical and active SAR systems as well as to solve multisensor/multisource data problems. This becomes even more true when distribution-free non-parametric classifiers are considered.

Unfortunately, in several situations and applications ground truth information cannot be collected or the process becomes too expensive. In such situations, unsupervised methods become the only opportunity. This is the reason why the scientific community is still very active on this topic even if the literature is extensive. Once multitemporal images have been radiometrically and geometrically corrected, unsupervised change detection information extraction requires mainly 2 steps: i) image comparison, that results in a change feature (CF). This step aims at highlighting the presence of change and accounts for the temporal correlation among acquisitions; and ii) analysis of the change feature. This step aims at isolating the change from the no change information. The first step is the one that mostly depends on the kind of considered data.

When dealing with optical passive sensor images, comparison mainly relies on the difference operator. This is because the noise model in optical images is additive and the natural classes tend to have a Gaussian distribution. The simplest way to use the difference operator is to apply it to one or multiple corresponding spectral bands from multitemporal images [66]leading to the definition of Spectral Change Vectors (SCVs). The latter option is referred to as Change Vector Analysis and has been effectively employed with multispectral and hyperspectral images, and low to high resolution images as well $[12,19,16,54,55]$. Under the assumption of Gaussian distributed natural classes and being the difference a linear operator, classes of change and no-change in the SCV feature space result to be Gaussian distributed as well [12]. Non-linear features are commonly extracted from SCVs [12, 55, 54, 73] like the magnitude and direction variables. The magnitude of changed samples presents significantly higher values than those of pixels associated to unchanged areas $[19,66]$. Thus, the magnitude allows for a simple binary detection between change and no-change. On the other side, the direction variable is highly relevant to distinguish among different kinds of changes as they assume preferred directions $[12,55,54,66]$. The difference operator can be applied in feature spaces other than the original spectral band one. Examples can be found that apply it to posterior probabilities [28], vegetation indexes [66], Tasselled Cap Transformation features 
[67], Multivariate Alteration Detection features [60], non-linear combinations of spectral bands, etc.

\subsection{SAR change detection}

When dealing with SAR images the commonly accepted noise model is multiplicative. Under this assumption, it is possible to show that after subtraction the statistical distribution of the resulting image depends on both the relative change between the intensity values in the two images and a reference intensity value (i.e., the intensity before or after the change). This leads to a higher change-detection error for changes occurred in high-intensity regions of the image than in low-intensity regions. Thus the ratio operator (Image Rationing) [66] is more indicated for SAR multitemporal image comparison since its distribution depends only on the relative change in the average intensity between the two dates and not on a reference intensity level $[61,8]$. Further, it allows to reduce common multiplicative error components[61]. In the literature, the ratio image is usually expressed in a logarithmic scale. Thus the log-ratio operator is typically preferred $[8,30,36,61,63]$. Another set of comparison operators widely used with SAR (but valid for optical data as well [58]) is the one based on the use of information theoretical similarity measures: the Kullback-Leibler (KL) divergence [48]; the Mutual Information [4]; and combinations of them. More recently the multi-scale/-resolution concept has been introduced in the multitemporal image analysis. This need emerged because of the complexity of SAR data and because of the intrinsic multiresolution information available in the images acquired by the new generation high spatial resolution sensors. To properly model multi-scale/-resolution information different approaches have been used. Among the others we recall the Wavelet decomposition [11, 27], the Contourlet transform [52], and multiscale feature profiles computed on varying windows size (Sect. 2.3.3), multiscale segments [10, 45], and morphological profiles [35, 44]. More sophisticated approaches to the representation of multiresolution information have been developed when very high spatial resolution (VHR) images are analysed. They model the high level semantic information in VHR images at a higher level of abstraction [14, 16, 50] and thus become intrinsically suitable for multisensor analysis [15].

The typical methodological approach for SAR images considers a direct pixelbased comparison of the two images, that generates a change feature (CF), which is taken as input for the decision step. Typically, many approaches in the literature consider an unsupervised thresholding of CF [4],[27]. Nevertheless, the analysis is affected by the multiplicative speckle noise present in the SAR images and its compensation implies a degradation in terms of spatial resolution. In order to deal with this issue, $\mathrm{CD}$ methods were designed that achieve different trade-offs in terms of both accuracy by the compensation of the speckle effect and preservation of the high-resolution geometrical information.

Scale-driven analysis [11],[48], among them, considers different scale levels in the $\mathrm{CD}$ analysis. It is based on the multiscale decomposition of the CI image, 
on the selection of the reliable scales for each pixel and subsequent image fusion and decision. In particular, the multiscale decomposition considers the use of twodimensional filtering (e.g., stationary wavelet transform) on the image which applies, in both row-wise and column-wise, either low-pass or high-pass filtering.

\subsection{Change Detection Methods for VHR SAR Images}

Concerning VHR remote sensing images, the high geometrical information content requires an accurate definition and modeling of the concept of change which is often associated with the specific goal of the application. The complexity is increased by the need to take into account all the specific issues related to the properties of VHR data. Standard unsupervised change-detection techniques in the remote sensing literature often do not perform a detailed analysis of the concept of change. Usually they compare two images acquired on the same geographical area at different times by assuming that their radiometric properties are similar except for the presence of changes occurred on the ground [16].

When application-oriented prior information is not available, change can be detected from the image radiometric properties only. For VHR amplitude SAR images, a single scale approach is rarely effective, while a multiscale approaches can improve the detection performance through the analysis of different scales of representation of the change signal, where each scale is characterised by a different trade-off between speckle reduction and preservation of geometrical details [11].

Let us consider two SAR images $X_{k}$, of size $I \times J$, acquired over the same geographical area at two different times $t_{k}$, with $k=1,2$. Let us assume that the bi-temporal images are co-registered, geo-referenced and radiometrically corrected. Let $\omega_{n c}, \omega_{c}$ be the set of classes associated with unchanged and changed pixels, respectively.

Here, we investigate the capabilities of both single-scale and multiscale approaches in detecting changes in bi-temporal SAR acquisitions $X_{1}$ and $X_{2}$. Three selected algorithms are described in the following subsections.

\subsubsection{Information-theoretic feature}

The mean-shift information-theoretic change detection (MS-ITCD) method relies on a feature capturing the structural change between $X_{1}$ and $X_{2}$. It is robust to the statistical change that may be originated by speckle and co-registration inaccuracies. The method starts from the scatterplot of the amplitude levels in the two images and applies the mean-shift (MS) algorithm to find the modes of the underlying bivariate distribution [4]. 
The rationale of the algorithm is that the negative of the logarithm of the probability of a mean amplitude level in one image conditional to the mean amplitude level of the same pixel in the other image measures the amount of information associated to the pixel change and hence the amount of change, which may be related to the conditional information of couples of symbols emitted by two information sources [32].

Let $x_{1}(i, j)$ and $x_{2}(i, j)$ be the symbols emitted by the two information sources $X_{1}$ and $X_{2}$, respectively, where $i=0, \ldots I-1$ and $j=0, \ldots J-1$. The average information content of the two sources is given by their entropy, $H\left(X_{1}\right)$ and $H\left(X_{2}\right)$. In general, a part of such information is common to the two sources. This common information is called mutual information and is a measure of the statistical dependency between $X_{1}$ and $X_{2}$, i.e.,

$$
I\left(X_{1} ; X_{2}\right)=H\left(X_{1}\right)-H\left(X_{1} \mid X_{2}\right)
$$

or, equivalently,

$$
I\left(X_{1} ; X_{2}\right)=I\left(X_{2} ; X_{1}\right)=H\left(X_{2}\right)-H\left(X_{2} \mid X_{1}\right)
$$

where $H\left(X_{2} \mid X_{1}\right)$ is the conditional entropy of $X_{2}$ to $X_{1}$ and represents the fraction of $H\left(X_{2}\right)$ that cannot be inferred from the knowledge of the reference source $X_{1}$, because it is due to unpredictable changes.

Given the conditional information between $x_{2}(m, n)$ and $x_{1}(m, n)$, that is,

$$
I\left(x_{2}(i, j) \mid x_{1}(i, j)\right) \triangleq-\log \left[p\left(x_{2} \mid x_{1}\right)\right]
$$

the conditional entropy is the expected value of (3),

$$
H\left(X_{2} \mid X_{1}\right) \triangleq-\sum_{x_{1}} \sum_{x_{2}} p\left(x_{1}, x_{2}\right) \log \left[p\left(x_{2} \mid x_{1}\right)\right]
$$

where $p\left(x_{1}, x_{2}\right)$ and $p\left(x_{2} \mid x_{1}\right)$ are the joint probabilities of $x_{1}(i, j)$ and $x_{2}(i, j)$ and the conditional probabilities of $x_{2}(i, j)$ to $x_{1}(i, j)$, respectively.

The method, which features a fast version of Mean Shift (MS), and its earlier version are summarised in the following procedure:

1. Given two co-registered amplitude SAR images $x_{1}(i, j)$ and $x_{2}(i, j)$ taken on the same scene at different times, estimate their local means at each pixel, $\bar{x}_{1}(i, j)$ and $\bar{x}_{2}(i, j)$, over a $(2 r+1) \times(2 r+1)$ sliding window with Gaussian weighting.

2. Cross-calibrate $\bar{x}_{2}$ over $\bar{x}_{1}$ by matching the global mean and variance of the former to those of the latter; hereafter, let $\bar{x}_{2}$ denote the histogram-matched version of $\bar{x}_{2}$.

3. Scale the values of both $\bar{x}_{1}$ and $\bar{x}_{2}$ by $\max _{m, n}\left\{\bar{x}_{1}, \bar{x}_{2}\right\}$; hereafter, let $\bar{x}_{1}$ and $\bar{x}_{2}$ denote the scaled version of the former $\bar{x}_{1}$ and $\bar{x}_{2}$. 
4. Draw the scatterplot of $\bar{x}_{2}(i, j)$ against $\bar{x}_{1}(i, j)$; thus, the scatterplot is contained in a square of unity side and unchanged pixels lie along its main diagonal.

5. Multiply $\bar{x}_{1}$ and $\bar{x}_{2}$ by an integer $L$, which will denote the size of the $2 \mathrm{D}$ histogram obtained from the binning of the scatterplot. Hereafter, $\left(\bar{x}_{1}, \bar{x}_{2}\right) \in\{[0, L] \times[0, L]\}$.

6. Calculate the $L \times L$ joint histogram $h(m, n), m=0, \ldots, L-1, n=0, \ldots, L-1$, by counting all points $\left(\bar{x}_{1}, \bar{x}_{2}\right)$ such that $n \cdot L<\bar{x}_{1} \leq(n+1) \cdot L$ and $m \cdot L<\bar{x}_{2} \leq$ $(m+1) \cdot L$.

7. Estimate the discrete joint probability density function (PDF), $p(m, n)=$ $p\left(\left\lfloor\bar{x}_{2}\right\rfloor,\left\lfloor\bar{x}_{1}\right\rfloor\right)$, by normalizing $h(m, n)$ to the overall number of points and convolving it by a normalised triangular kernel of length $(2 t+1)$, according to Parzen window method.

8. Divide $p(m, n)$ by its maximum along $\bar{x}_{1}, \max _{j} p(m, n)$, so that its value is one, and hence the logarithm is zero (no change) when $p(m, n)$ attains its maximum over $j$ :

$$
q(m \mid n)=\frac{p(m, n)}{\max _{j} p(m, n)}=p(m \mid n) \cdot \frac{p(n)}{p_{\max _{n}}(m)} .
$$

9. Pre-calculate a LookUp Table (LUT) of the information-theoretic change detection (ITCD) feature for each pair of $(i, j)$ that indexes $q(i \mid j)$ as:

$$
C(m, n)=-\log \{q(m \mid n)\}
$$

10. To calculate a map of plain ITCD feature, for each pixel $(i, j)$, calculate $\bar{x}_{1}=$ $\bar{x}_{1}(i, j)$ and $\bar{x}_{2}=\bar{x}_{2}(i, j)$, as in Step 5, then $\operatorname{ITCD}(i, j)=C\left(\left\lfloor\bar{x}_{2}\right\rfloor,\left\lfloor\bar{x}_{1}\right\rfloor\right)$.

11. To calculate a map of MS-enforced ITCD (MS-ITCD) feature, the MS clustering algorithm is applied to the "binned" scatterplot obtained at the end of Step 5, with a uniform kernel of radius $R$,

- perform migration of the scatterpoints belonging to an original bin $(m, n)$ : start from the center of the bin and move all scatterpoints at the same time toward the center of the attracting cluster.

- Let $(\mathbf{m}, \mathbf{n})$ denote the integer valued stop coordinates of MS applied to the bin $(m, n)$; the change feature $C(\mathbf{m}, \mathbf{n})$ is associated to all scatterpoints originally belonging to $(m, n)$;

- for each pixel $(i, j)$, calculate the bin $(m, n)$ in which the pixel falls, replace $(m, n)$ with $(\mathbf{m}, \mathbf{n})$ found through MS, set MS-ITCD $(i, j)=C(\mathbf{m}, \mathbf{n})$.

The effect of MS is moving the scatterpoints contained in each bin towards the attracting center corresponding to a mode of the underlying PDF, as defined at Step 7. Unchanged pixels produce scatterpoints that are likely to be moved toward one of the modes along the main diagonal; conversely, changed pixels will be moved towards one of the modes far from the main diagonal.

The main difference of the MS-enforced ITCD, originally introduced in [3] from its earlier version, ITCD, [5, 6, 2], is that the presence of MS makes the novel feature to follow a clustered approach: the information-theoretic feature is calculated from the values of conditional probabilities roughly corresponding to the modes of the joint PDF. 
The resulting MS-ITCD feature is considered and tested here in two configurations: as an example of high-performance single-scale change feature, and as the change feature adopted by the multiscale strategy described in the next Section.

\subsubsection{Multiscale CD strategy based on wavele decomposition}

A detailed description of the scale-driven $\mathrm{CD}$ approach, originally presented in [11], is provided in this section. The technique takes as input $X_{1}$ and $X_{2}$ and consists in four main steps: i) change features extraction by means of image comparison; ii) multiresolution decomposition; iii) adaptive scale identification on the basis of local statistics of both the full and lower resolution data; iv) adaptive fusion based on the optimal scale level and generation of the final CD map. A general block scheme of the approach is represented in Fig. 1.

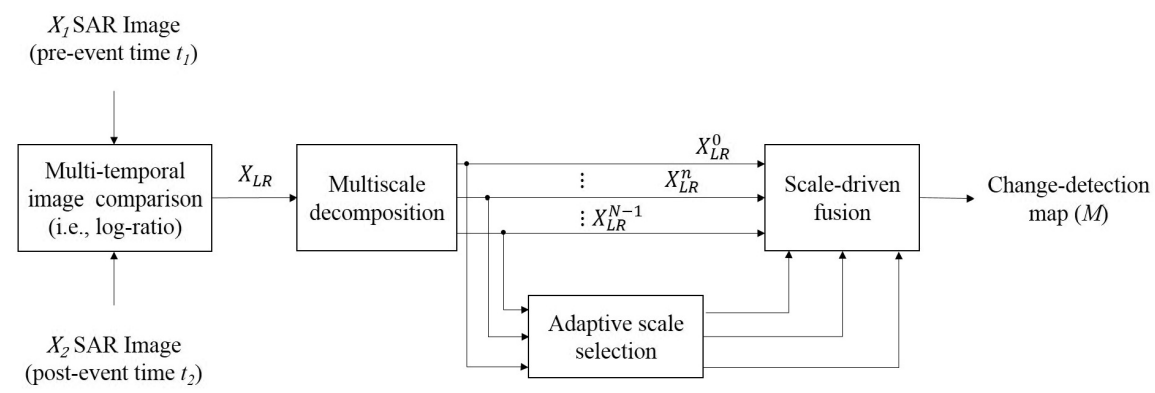

Fig. 1 Block scheme for the wavelet-based approach

In the first step image comparison is performed to compute a Change Feature (CF) that highlight backscattering variations. Among the operators presented in the literature, the log-ratio $\mathrm{X}_{L R}$ (see eq. (7)) is used here to illustrate the method [11, 8, 64]. However the method can be applied to other CFs as well (e.g., ratio [7], KL divergence [48], difference). Accordingly, in the experiments results will be illustrated both on the log-ratio image and on the ITCD feature.

$$
X_{L R}=\log \frac{X_{2}}{X_{1}}=\log X_{2}-\log X_{1} .
$$

This choice allows to reduce the effect of the speckle noise and to have a statistical distribution of the CF centered on the zero value, with the two classes of interest assuming each a more symmetrical distribution.

The multitemporal information in the $X_{L R}$ is still affected by residual undesired speckle. Therefore, the second step aims at reducing the residual speckle effect on the log-ratio image, while preserving the geometrical details. For this reason, a decomposition of the log-ratio image at different scale levels is computed by 
creating a set of images $X_{m s}=\left\{X_{L R}^{0}, \ldots, X_{L R}^{N-1}\right\}$, where $X_{L R}^{n}$ indicates the $n$-th level decomposition level, $n=0,1, \ldots, N-1$. To this end, a dyadic decomposition is applied, so that the scale corresponding to each resolution level is given by $2^{n}$ and the image for $n=0$ corresponds to the original log-ratio image. Among the possible approaches presented in the literature for the two-dimensional image decomposition, such as Laplacian pyramid decomposition [47] or recursively upsampled bicubic filter [41], we consider the Two-Dimensional Stationary Wavelet Transform (2DSWT), by following [11, 27].

This filtering approach applies level-dependent filters to the considered signal at each resolution level, by working separately along rows and columns, respectively. Typical filters for this kind of applications are 4th-order Daubechies filters.

This approach presents the advantage of avoiding down-sampling and possible aliasing impairments. At each step of the 2D-SWT, the image of the low-resolution components is taken as input and filtered, both row-wise and column-wise, with lowpass and high-pass filters, in order to separate lower resolution components $(L L)$ and detail components on vertical $(L H)$, horizontal $(H L)$ and diagonal $(H H)$ direction, which are defined as:

$$
\begin{aligned}
& X_{L R}^{L L(n+1)}(i, j)=\sum_{p=0}^{D^{n}-1} \sum_{q=0}^{D^{n}-1} l^{n}[p] l^{n}[q] X_{L R}^{L L(n)}(i+p, j+q) \\
& X_{L R}^{L H(n+1)}(i, j)=\sum_{p=0}^{D^{n}-1} \sum_{q=0}^{D^{n}-1} l^{n}[p] h^{n}[q] X_{L R}^{L L(n)}(i+p, j+q) \\
& X_{L R}^{H L(n+1)}(i, j)=\sum_{p=0}^{D^{n}-1} \sum_{q=0}^{D^{n}-1} h^{n}[p] l^{n}[q] X_{L R}^{L L(n)}(i+p, j+q) \\
& X_{L R}^{H H(n+1)}(i, j)=\sum_{p=0}^{D^{n}-1} \sum_{q=0}^{D^{n}-1} h^{n}[p] h^{n}[q] X_{L R}^{L L(n)}(i+p, j+q)
\end{aligned}
$$

At level 0 , the low-resolution component corresponds to the original log-ratio image. The filter coefficients at level $n+1$ are obtained with a dilation of the coefficients of the filter at level $n$ by a factor of 2. After the decomposition and by skipping the high resolution components, the approximation images at each scale level are retrieved by applying the inverse two-dimensional stationary wavelet (2DISWT) transform. Because of both the assumption on the additive noise model in the logarithmic scale and the computational cost of the processing, the wavelet strategy is applied directly on the log-ratio image, generating the set of images $X_{m s}=\left\{X_{L R}^{0}, \ldots, X_{L R}^{N-1}\right\}$.

Each of the wavelet output is used for generating a corresponding CD map, where the thresholds can be selected either manually or automatically, i.e., Bayesian approach based on the EM algorithm (see also Chapters 4, 5, and 9), or KittlerIllingworth thresholding which is a computationally efficient solution to the problem of minimum error thresholding for normally distributed variables. 
The final step of the algorithm is the fusion of the information in the products at different wavelet scales and the generation of the final CD map. Different fusion strategies are available in the literature, applying the fusion at the decision level and considering different choices for the thresholds. Fusion at the feature level on all reliable scales (FFL-ARS) [11] considers a reliable scale depending on the individual pixel and operates a fusion at feature level. Conversely, the fusion at the decision level on all reliable scales (FDL-ARS) [11] still considers a reliable scale depending on the individual pixel, but it operates the fusion at the decision level. The literature has proven that the best performance in terms of overall accuracy is obtained by the FFL-ARS approach, because of the best trade-off between the reduction of the speckle level and the details preservation. This fusion strategy is based on the generation of a new set of images, namely $\bar{X}_{m s}=\left\{\bar{X}_{m s}^{0}, \ldots, \bar{X}_{m s}^{N-1}\right\}$, derived from $\mathrm{X}_{m s}$, in which each image $\bar{X}_{m s}^{n}$ is computed as an average of the wavelet decomposition up to the level $n$, as described in (12):

$$
\overline{\mathbf{X}}_{\mathrm{ms}}^{n}=\frac{1}{n+1} \sum_{h=0}^{n} \mathbf{X}_{\mathrm{LR}}^{h}, \quad n=0,1, \ldots, N-1
$$

In the FFL-ARS approach, for each pixel, reliable scale levels are determined according to whether the considered pixel belongs to either an edge or a nonhomogeneous region. In particular, for each of the scales it evaluates two coefficients: a global coefficient of variation $\left(C V^{n}\right)$, defined on the whole image, and a local coefficient of variation $\left(L C V^{n}(i, j)\right)$, defined on sliding window of user-defined size centered on the pixel $(i, j)$.

These coefficients are expressed as:

$$
\begin{aligned}
L C V^{n}(i, j) & =\frac{\sigma^{n}(i, j)}{\mu^{n}(i, j)} \\
C V^{n} & =\frac{\sigma^{n}}{\mu^{n}}
\end{aligned}
$$

The coefficient of variation cannot be computed on the multiscale log-ratio images, so the computation of these two coefficients is done on the multiscale ratio image sequence, derived by inverting the logarithm operation for each of the scale levels. For a general pixel $(i, j)$, the decomposition scale $R_{i j}$ is defined as reliable if the following condition is satisfied for all the resolution levels $\left(l=0,1, \ldots, R_{i j}\right)$ :

$$
\begin{gathered}
M(i, j) \in \omega_{k} \Leftrightarrow M^{R_{i j}}(i, j) \in \omega_{k}, k \in\{c, n c\}, S_{i j} \leq N-1 \\
M^{t}(i, j) \in \omega_{k} \Leftrightarrow L C V^{t}(i, j) \leq C V^{t}
\end{gathered}
$$


The final CD map is obtained from the set of multiresolution maps by applying a standard thresholding procedure to the fused images and recombining them by selecting the most reliable scale level for each pixel, as

$$
M(i, j)= \begin{cases}\omega_{n c} & \text { if } x=\bar{X}_{m s}^{R_{i j}}(i, j) \leq T^{R_{i j}} \\ \omega_{c} & \text { if } x=\bar{X}_{m s}^{R_{i j}}(i, j)>T^{R_{i j}}\end{cases}
$$

where $T^{R_{i j}}$ is the decision threshold optimised for the considered fused image $\bar{X}_{m s}^{R_{i j}}$ and $(i, j)$ is the spatial position of the considered pixel.

For the set $\overline{X_{m s}}$, the value at the reliable scale $\bar{X}_{m s}^{R_{i j}}(i, j)$ and the related threshold $T_{m s}^{R_{i j}}(i, j)$ are associated for each pixel $(i, j)$. As described above, the threshold values, derived for the different wavelet levels, can be either manually or automatically set, according to any of the different strategies in the literature.

\subsubsection{Combination of multiscale change features}

Let $r_{w}$ denote the bounded ratio image $\left(0<r_{w}<1\right)$ computed from two coregistered amplitude SAR images $X_{1}$ and $X_{2}$ acquired on the same scene at different dates:

$$
r_{w}=\min \left\{\frac{\bar{X}_{1}^{(w)}}{\bar{X}_{2}^{(w)}}, \frac{\bar{X}_{2}^{(w)}}{\bar{X}_{1}^{(w)}}\right\},
$$

where $\bar{X}_{k}^{(w)}$ indicates the $k$-th image averaged over a $w \times w$ sliding window.

The bounded ratio image is computed for different odd window sizes in the interval $S=\left[w_{\min }, w_{\max }\right]$, and the resulting $N_{w}=\left(w_{\max }-w_{\min }\right) / 2+1$ multiscale features are finally combined into the single geometric-mean bounded-ratio (GMBR) change feature $R_{S}$ :

$$
R_{S}=\left(\prod_{w \in S} r_{w}\right)^{1 / N_{w}}
$$

The GMBR feature $R_{S}$ has the important property of being intrinsically normalised, which is convenient for unsupervised clustering, it is easy to compute, robust to speckle impairments, and shows good capability of spatial detail preservation, thanks to its multiscale nature.

The interval $S$ of the window sizes should be selected according to the number of looks of the SAR images (or equivalently, their resolution), and possibly to the expected size of the regions of change. Typical values are $S=[5,25]$ for 1-look data and $S=[3,11]$ for 4-look data.

The GMBR feature can be properly clustered into the two classes of unchanged pixels, $\omega_{n c}$, and changed pixels, $\omega_{c}$, by applying the unsupervised K-means al- 
gorithm with $K=2$. An example of $R_{S}$ is reported in Fig. 4(b) and the resulting change map obtained by K-means clustering is shown in Fig. 10(d).

\section{Experimental Results}

This section presents the experimental results obtained on two different datasets, a simulated and a real one. In the next subsections, each dataset is described and the performance analysis on four different change detection strategies is derived:

1. The MS-ITCD feature is first tested with optimal window size $w$ and radius $R$ (i.e., $w=9$ pixels, $R=30$ quantised amplitude levels) for best change mapping performance (see steps 1 and 11 of the MS-ITCD algorithm) by means of two-class k-means clustering.

2. The GMBR feature is computed and then clustered for change mapping.

3. The wavelet-based approach adopting the FFL-ARS fusion strategy driven by the log-ratio change feature, referred as FFL-ARS1 (see Fig. 1), is tested.

4. The FFL-ARS fusion strategy driven by the MS-ITCD feature with smaller window and radius ( $w=5$ pixels, $R=10$ quantised amplitude levels), referred as FFL-ARS2, is finally applied.

\subsection{Simulated data}

We have considered two different datasets of SAR images, on which different change events were simulated. In particular, these changes are related to four regions of backscattering increase and one of backscattering decrease. Two synthetic image pairs with known patches with different shapes, sizes and change levels have been produced from an optical remote sensing image, a panchromatic Ikonos image of Toulouse, France, with 12-bit dynamic range. Nakagami-distributed speckle patterns [1] have been generated with different equivalent number of looks $L$ and spatial correlation values $\rho_{s}$, specifically, $L=1$ and $\rho_{s}=0.3$ for the first dataset, and $L=4$ and $\rho_{s}=0$ for the second dataset. It should be recalled that Nakagami-distributed speckle in the SAR amplitude domain is equivalent to Gamma-distributed speckle in the SAR intensity domain (see also Chapters 4 and 5). The first dataset has $1 \mathrm{~m}$ spatial resolution and $1 \mathrm{~m}$ pixel spacing, thus simulating a CSK Enhanced Spotlight image pair, while the second dataset simulates Sentinel-1 Stripmap Mode images having $9 \mathrm{~m}$ spatial resolution and $4 \mathrm{~m}$ pixel spacing.

The two datasets represent the same geographical area of about $0.5 \mathrm{~km}^{2}$, through a $720 \times 720$ image pair for the 1-look dataset (Fig. 2), and a $180 \times 180$ image pair for 


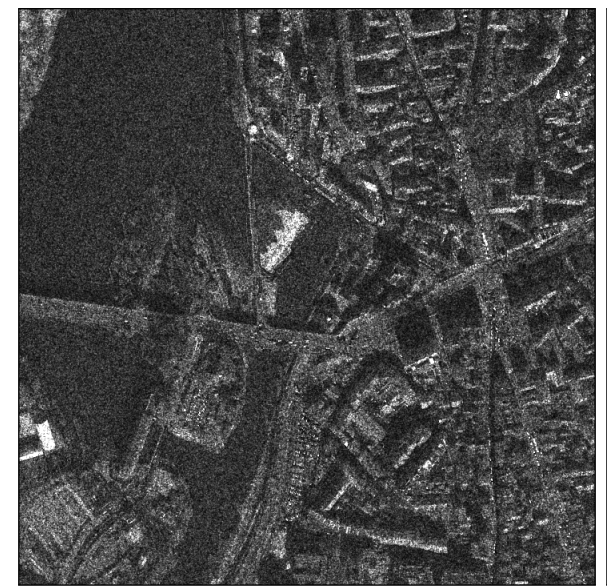

(a)

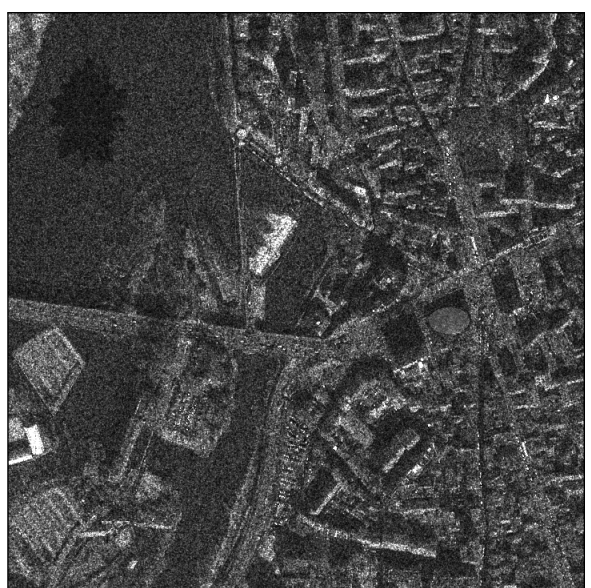

(b)

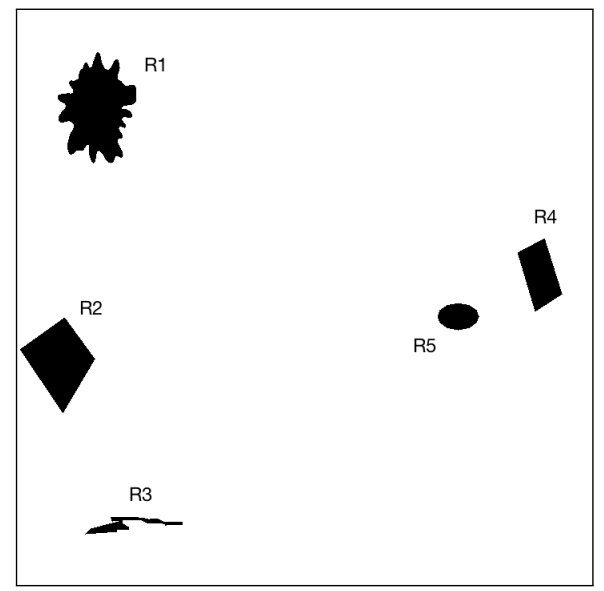

(c)

Fig. 2 (a), (b): Simulated $720 \times 720$ 1-look image pair; ground truth change image (c).

the 4-look dataset (Fig. 3). The simulated change patches are regions with modified backscattering, specifically a $30 \%$ reduction of the amplitude level in the second date with respect to the first date in the $\mathrm{R} 1$ region, deterministic cover changes through pasting image values in the R2, R3 and R4 regions, and a constant increase of 80 amplitude levels on the R5 region (see Fig. 2(c); corresponding regions are represented in Fig. 3(c)). 


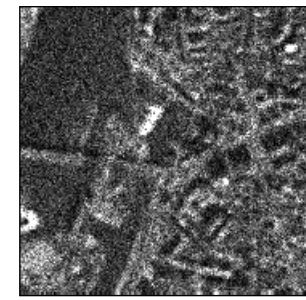

(a)

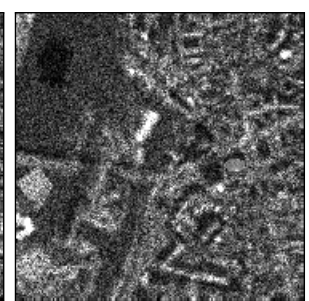

(b)

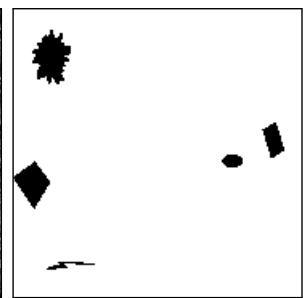

(c)

Fig. 3 (a), (b): Simulated $180 \times 180$ 4-look image pair; ground truth change image (c).

\subsubsection{Quantitative Performance Assessment}

The four change detection strategies are first compared in terms of the Receiver Operator Characteristics (ROC). MS-ITCD and GMBR are directly compared in order to give evidence to the different characteristics of single-scale MS-ITCD and multiscale GMBR features (Fig. 4,5).

FFL-ARS1 and FFL-ARS2 are not reported in the same graph since they cannot be considered as CD features, but as fusion strategies of multiple wavelet features. As previously stated, the FFL-ARS1 strategy relies on the log-ratio computation, while FFL-ARS2 is based on the MS-ITCD change index.

On the other hand, all the four CD methods can be straightly compared in terms of final binary CD maps, as reported in Sect. 3.1.2.

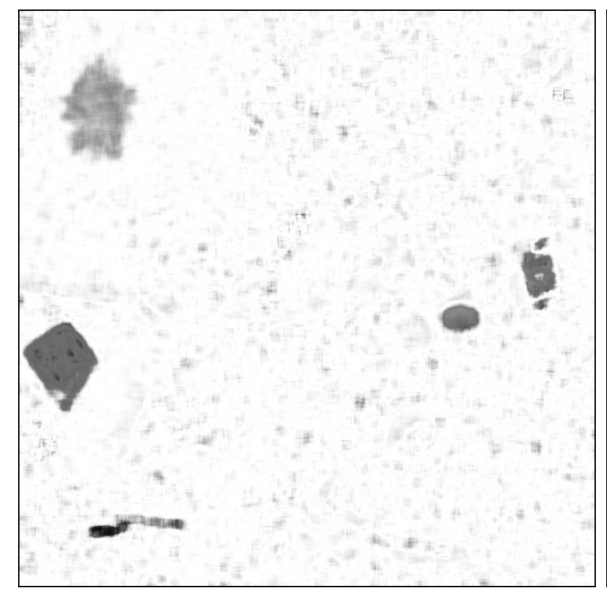

(a)

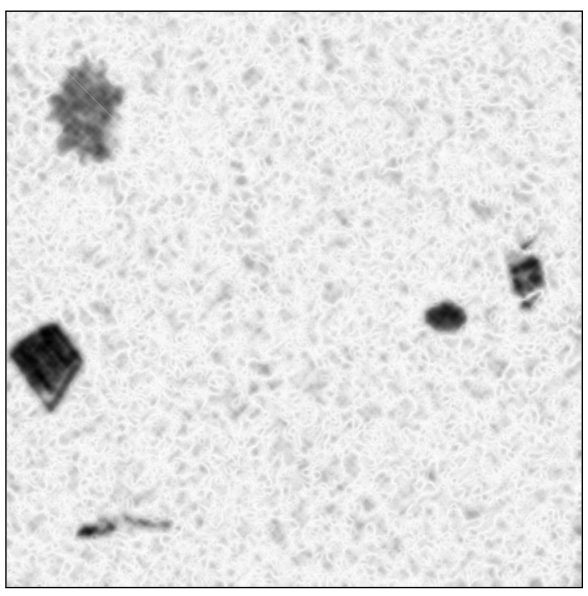

(b)

Fig. 4 Change features for the 1-look image pair: MS-ITCD (a); GMBR (b).

Fig. 6 shows that MS-ITCD outperforms GMBR only for high values of False Positive Rate (FPR). However, since the optimal change maps, corresponding to the 


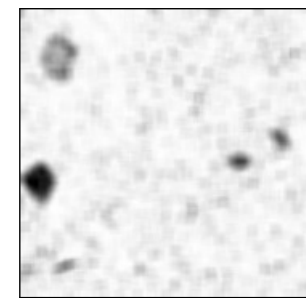

(a)

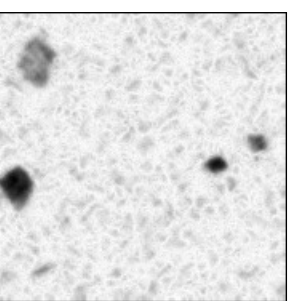

(b)

Fig. 5 Change features for the 4-look image pair: MS-ITCD (a); GMBR (b).

highest values of the Cohen's kappa coefficient (20), are obtained for lower values of FPR, as evidenced by Fig.7, the best mapping performance for both 1-look and 4-look image pairs are provided by GMBR.

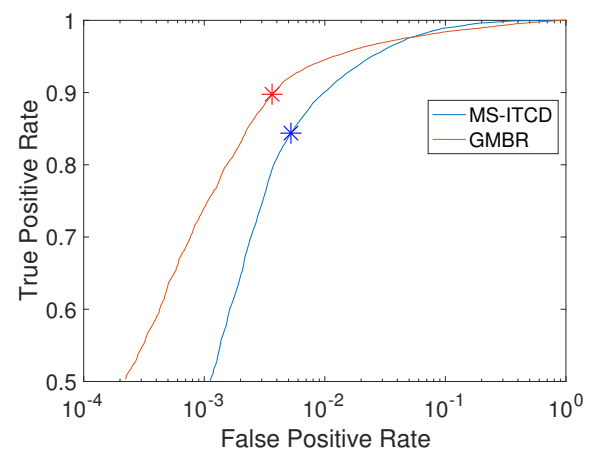

(a)

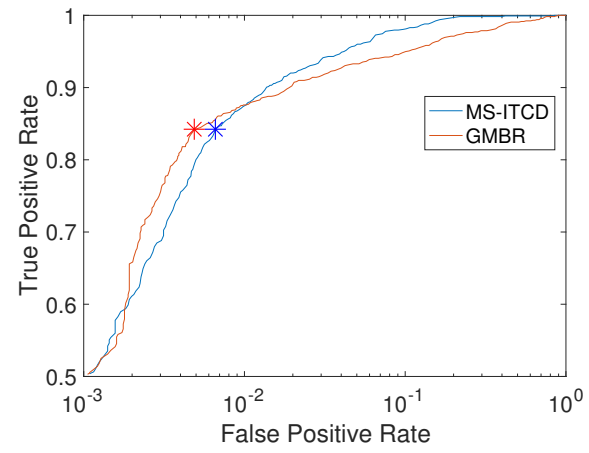

(b)

Fig. 6 ROC of the MS-ITCD and GMBR change features for the 1-look (a) and 4-look (b) simulated data. The asterisks highlight the optimal operating points (see also Fig. 7). 
By comparing Figs. 6(a) and 6(b), the GMBR ROC curve for the 1-look case is quite surprisingly higher than the ROC curve for the 4-look case (red curves in the two figures). This is due to the characteristics of GMBR which is more sensitive to the degradation of the spatial resolution rather than to the signal-to-noise reduction due to speckle.

For FFL-ARS1 and FFL-ARS2 experiments ROC curves obtained at different wavelet decomposition levels are reported in Fig. 8 and Fig. 9, respectively. In order to have a suitable number of pixels at all decomposition levels, maximum decomposition levels $N=5$ and $N=3$ have been considered for the analysis of the 1-look and the 4-look image pairs, respectively.
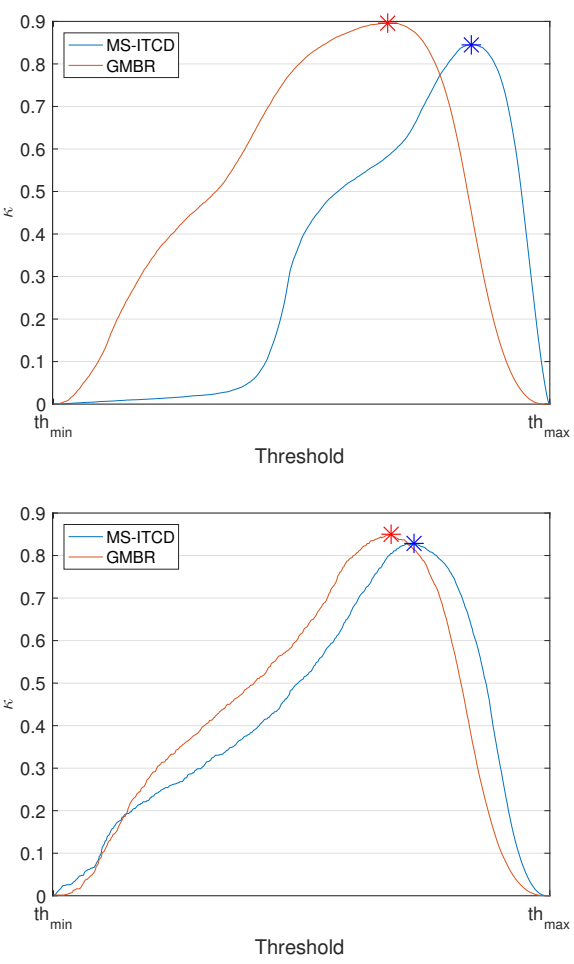

Fig. 7 Cohen's kappa of the feature-based change map with respect to the true change map as a function of the threshold applied to each feature. Top: 1-look; down: 4-look simulated data. The asterisks indicate the maximum values obtained by applying k-means clustering with $K=2$ to GMBR (in red) and MS-ITCD (in blue).

For the FFL-ARS1 strategy, the set of images $\bar{X}_{m s}$, i.e., starting from the log-ratio image, has been derived. Each image in $\bar{X}_{m s}$ has been separately thresholded and the CD performance of each decomposition level has been evaluated by tracing ROCs. Fig. 8 shows the ROC curves obtained at the different wavelet decomposition levels of 


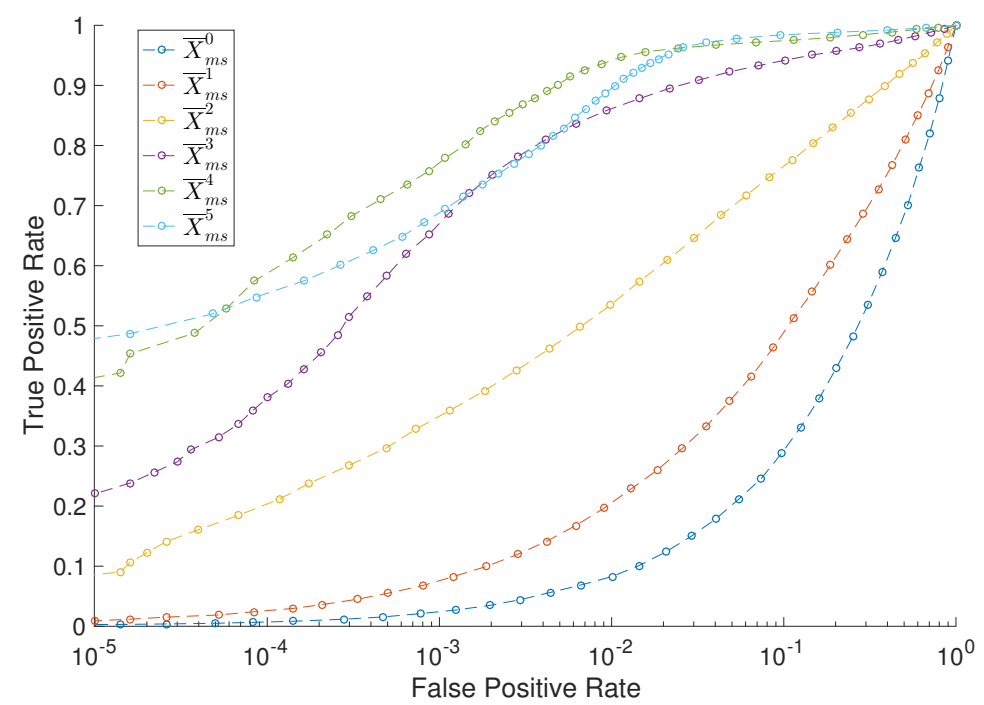

(a)

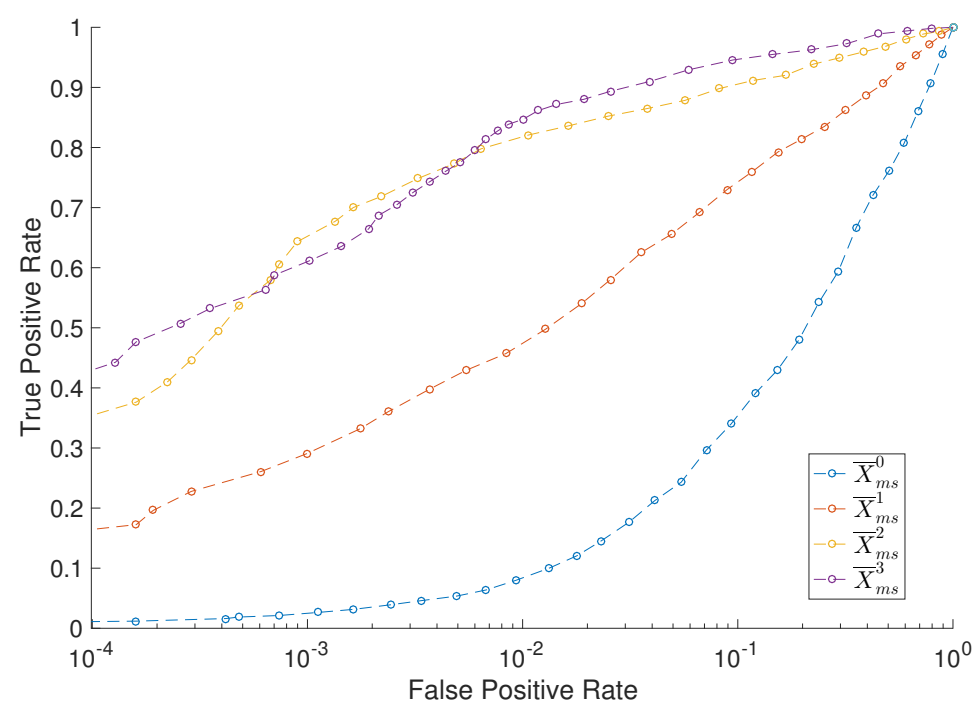

(b)

Fig. 8 ROC of the multiscale change feature used by FFL-ARS1 for the 1-look (a) and 4-look (b) simulated data.

the log-ratio feature. It should be noted that for the single-look case in Fig. 8(a), as the wavelet decomposition level $n$ increases, the ROC curves show higher True Positive Rate (TPR) and better CD performance, with an optimal number of decomposition 


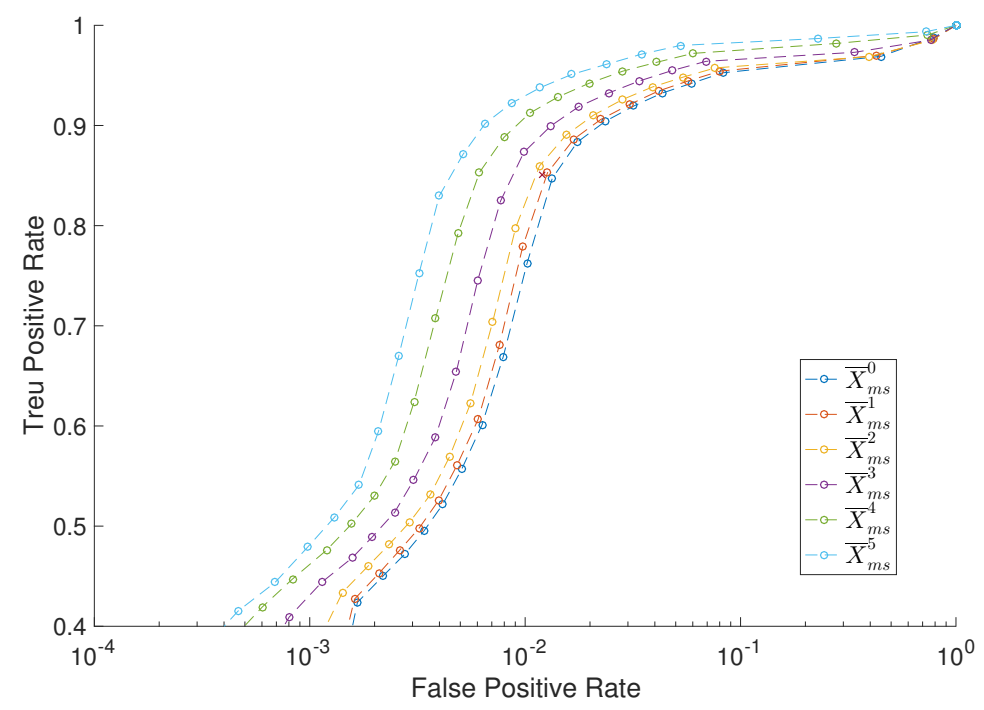

(a)

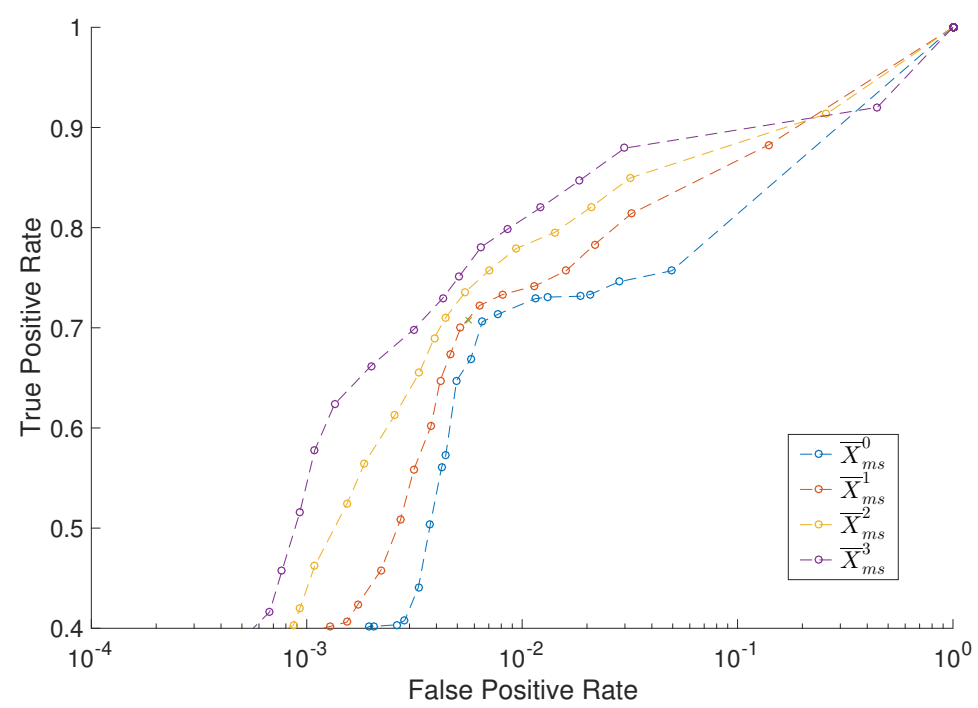

(b)

Fig. 9 ROC of the multiscale change feature used by FFL-ARS2 for the 1-look (a) and 4-look (b) simulated data.

levels equal to 4 . This can be explained by the inclusion of the low resolution information in the average products $\bar{X}_{m s}$. In this image components, the speckle effect is mitigated and classification over homogeneous areas is improved. 
At the highest decomposition level $N=5$ this trend is not confirmed, due to an extreme degradation of the spatial resolution. Fig. 8(b) shows that, for the 4-look case, by using only 2 or 3 levels of decomposition, depending on the required false positive rate, we get good $\mathrm{CD}$ performance. This is because of the loer geometric resolution of the 4-look image with respect to the 1-look one. Thus higher level of decomposition show a too low geometrical resolution, with a degradation of the overall CD capabilities.

Concerning the FFL-ARS2 strategy, the set of images $\bar{X}_{m s}$, i.e., starting from the MS-ITCD feature, has been derived. Again, each $\bar{X}_{m s}$ image has been separately thresholded to produce a ROC curve. Fig. 9 shows the ROC curves obtained at different wavelet decomposition levels by this method. For the single-look case in Fig. 9(a), as the wavelet decomposition $n$ increases, the ROC curves show higher TPR and better CD performance, with best performance for $N=5$. Similarly to the FFL-ARS1 strategy, also for the FFL-ARS2 approach Fig. 9(b) shows that, for the 4-look case, 2 or 3 levels of decomposition provide the best performance, depending on the required false positive rate. Therefore, the FFL-ARS algorithm can benefit from the different characteristics of the $\mathrm{CD}$ features at different scales.

Since the ground truth is available for the two simulated scenarios, the confusion matrix $C$ can be computed to provide a quantitative performance assessment. The columns of the matrix represent the instances in the predicted classes $\left(\omega_{n c}^{\prime}\right.$, i.e., no change, in the first column, and $\omega_{c}^{\prime}$, i.e., change, in the second column), while the rows represent the instances in the true classes $\left(\omega_{n c}\right.$ or $\left.\omega_{c}\right)$.

Starting from the confusion matrix, it is possible to compute the Cohen's kappa which compares the accuracy of the classification system to the accuracy of a random system:

$$
\kappa=\frac{p_{o}-p_{e}}{1-p_{e}}=1-\frac{1-p_{o}}{1-p_{e}}
$$

where $p_{o}$ is the overall accuracy and $p_{e}$ is the accuracy of a random classifier. Differently from the confusion matrix, $k$ is a unique scalar value that provides a straightforward comparison among change maps obtained by different change detection algorithms.

For the 1-look image pair, we have:

$$
\begin{array}{ll}
C_{\mathrm{MS}-\mathrm{ITCD}}=\left[\begin{array}{cc}
497292 & 2337 \\
2696 & 16075
\end{array}\right] & C_{\mathrm{GMBR}}=\left[\begin{array}{cc}
498287 & 1342 \\
2114 & 16657
\end{array}\right] \\
C_{\mathrm{FFL}-\mathrm{ARS} 1}=\left[\begin{array}{cc}
497672 & 1957 \\
9412 & 9359
\end{array}\right] & C_{\mathrm{FFL}-\mathrm{ARS} 2}=\left[\begin{array}{cc}
493331 & 6298 \\
2478 & 16293
\end{array}\right]
\end{array}
$$

corresponding to the Cohen's kappa values reported in Table 1.

We recall that the MS-ITCD result has been obtained with optimal window size and radius ( $w=9$ pixels, $R=30$ quantised amplitude levels) for best change mapping performance by means of two-class k-means clustering, while the FFL-ARS2 fusion strategy on a $3 \times 3$ window has been driven by the MS-ITCD feature obtained with smaller window and radius, i.e., $w=5$ pixels and $R=10$ quantised amplitude levels. 
Table 1 Cohen's kappa values of the CD maps obtained from the 1-look image pair.

\begin{tabular}{lr}
\hline Algorithm & Cohen's kappa \\
\hline MS-ITCD & 0.860 \\
GMBR & 0.903 \\
FFL-ARS1 & 0.612 \\
FFL-ARS2 & 0.779 \\
\hline
\end{tabular}

For the 4-look image pair, we have:

$$
\begin{array}{rlrl}
C_{\mathrm{MS}-\mathrm{ITCD}} & =\left[\begin{array}{cc}
31025 & 198 \\
196 & 981
\end{array}\right] & C_{\mathrm{GMBR}}=\left[\begin{array}{cc}
31097 & 126 \\
223 & 954
\end{array}\right] \\
C_{\text {FFL-ARS1 }}=\left[\begin{array}{cc}
31154 & 69 \\
510 & 667
\end{array}\right] & C_{\mathrm{FFL}-\mathrm{ARS} 2}=\left[\begin{array}{cc}
31089 & 134 \\
318 & 859
\end{array}\right]
\end{array}
$$

corresponding to the Cohen's kappa values reported in Table 2.

Table 2 Cohen's kappa values of the CD maps obtained from the 4-look image pair.

\begin{tabular}{lr}
\hline Algorithm & Cohen's kappa \\
\hline MS-ITCD & 0.826 \\
GMBR & 0.840 \\
FFL-ARS1 & 0.689 \\
FFL-ARS2 & 0.798 \\
\hline
\end{tabular}

The best performance, i.e., the highest $\kappa$ values, are provided by the GMBR algorithm for both 1-look and 4-look data, thanks to an outstanding capability of rejecting false alarms with respect to FFL-ARS1 and, to a lesser extent, with respect to FFL-ARS2 and MS-ITCD. This advantage of GMBR is confirmed by Fig. 7, which also shows that the K-means clustering provides the optimal threshold values corresponding to the maximum values of $\kappa$.

Concerning the FFL-ARS method, detection performance depends on the choice of the window size parameter for the CV computation. In particular, for the FFLARS1 case, the overall performance increases, both in terms of $\kappa$ coefficient and misclassified pixels for large windows, while for FFL-ARS2 a small window size is preferred. In general, the FFL-ARS strategy aims at keeping the edge information of the changed regions. This is clear by observing the left central change in Fig. 10(d) when compared to the ground truth region R2 in Fig. 2(c).

The advantage of adopting a robust change feature such as MS-ITCD instead of the log-ratio image in the FFL-ARS fusion strategy is evident for both 1-look and 4-look data, as shown by numerical and visual results. 


\subsubsection{Qualitative Performance Assessment}

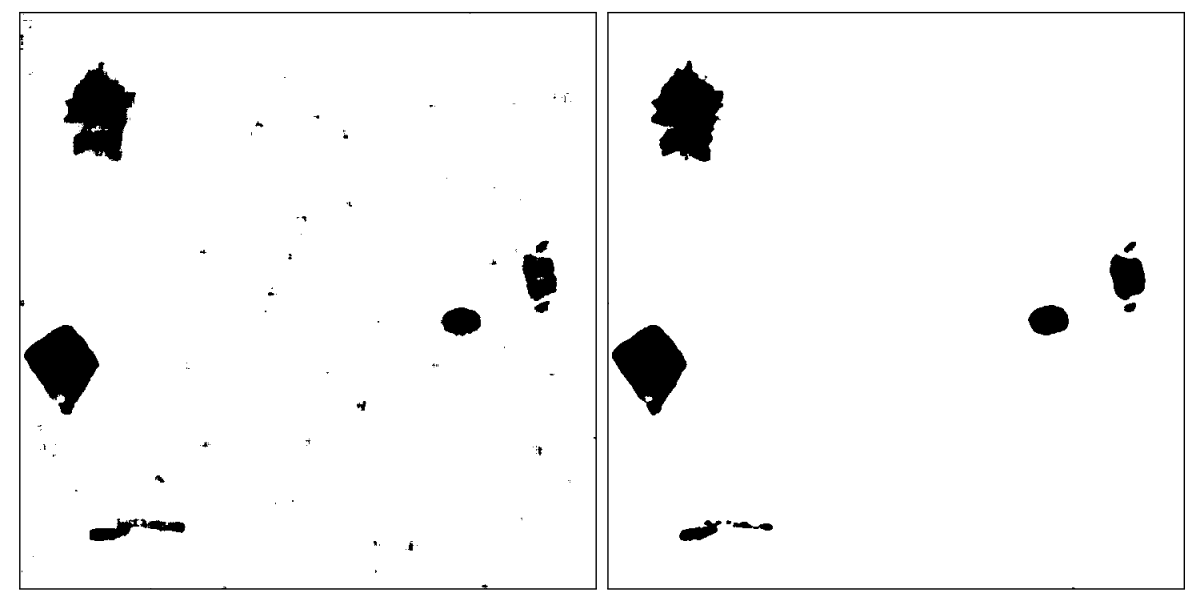

(a)

(b)

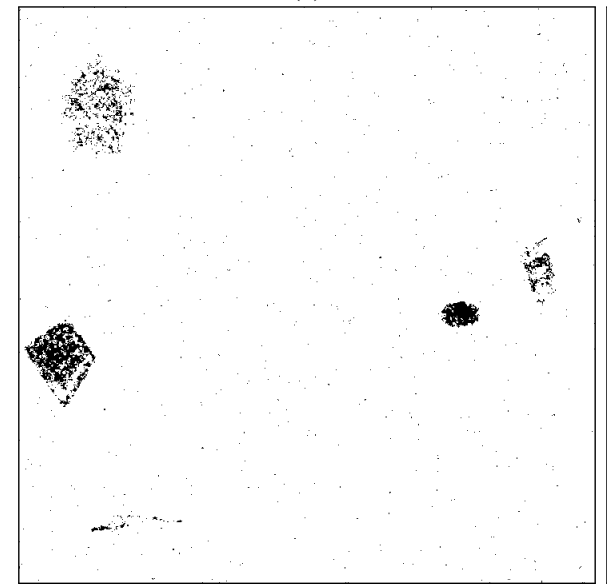

(c)

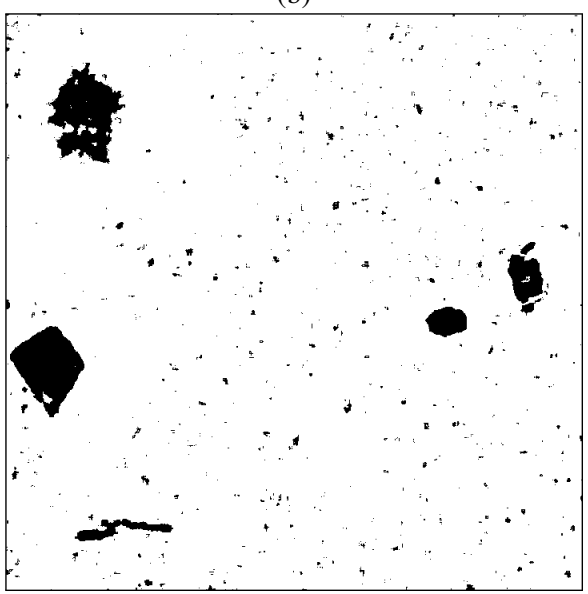

(d)

Fig. 10 Change maps for the 1-look case: MS-ITCD (a); GMBR (b); FFL-ARS1 (c); FFL-ARS2 (d).

Figures 10(a) and (b) show the final change maps computed by clustering, through $\mathrm{K}$-means with $\mathrm{K}=2$, the single-scale feature MS-ITCD (Sect. 2.3.1) and the multiscale feature GMBR (19), respectively. Both maps confirm the objective evaluation given by the confusion matrices and the $\kappa$ values, with excellent detection capabilities in the 1-look case. The multi-scale nature of GMBR also provides an outstanding false alarm rejection, as shown in Fig. 10(b). 
The comparison between the change detection maps obtained with FFL-ARS1 and FFL-ARS2 (with $w=5$ and $R=10$ ) (Fig. 10(c) and (d)) points out that the former one presents several misclassification errors, while the latter is much more accurate. An interesting characteristic of the FFL-ARS2 approach is its capability of preserving the spatial details of the changed regions, even better than the best performing GMBR algorithm.

Similar considerations apply for the change maps in the 4-look case reported in Fig. 11. The GMBR method provides the best change maps and shows very good false-alarm rejection, while MS-ITCD seems to suffer from the poor spatial resolution of 4-look data. FFL-ARS, in its MS-ITCD driven version, FFL-ARS2, can provide a good quality CD map, as in Fig. 11(d), although at the expense of an increased false alarm rate.

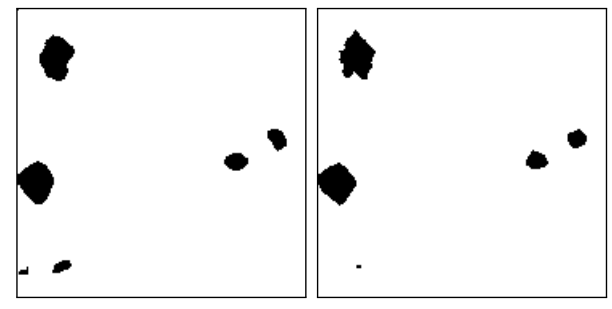

(a)

(b)

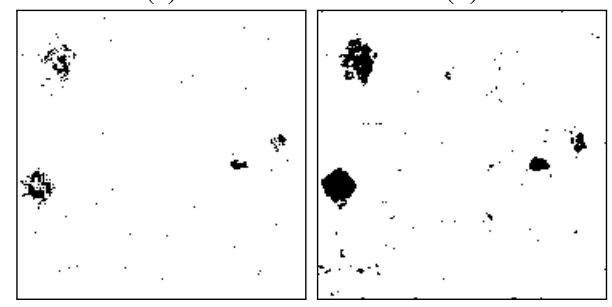

(c)

(d)

Fig. 11 Change maps from the 4-look image pair: MS-ITCD (a); GMBR (b); FFL-ARS1 (c); FFL-ARS2 (d).

\subsection{COSMO-Skymed Images}

For the real data set two COSMO-Skymed images have been considered and processed for assessing change detection capabilities in a true scenario. The considered change between the two acquisitions (April 5th, 2009 in Fig. 12(a) and September 12th, 2009 in Fig. 12(b), i.e., before and after the destructive earthquake on April 9th) is the construction of a tent camp set up for earthquake survivors near a shopping mall about $7 \mathrm{~km}$ West of the city center. Both 1-look acquisitions have been taken 
with right look-side, ascending pass, HH polarization, and 58 deg incidence angle. The two images have $1 \mathrm{~m}^{2}$ pixel size and are $1000 \times 1000$.

A manually generated ground truth of the tent camp built after the earthquake is reported in Fig.12(c).

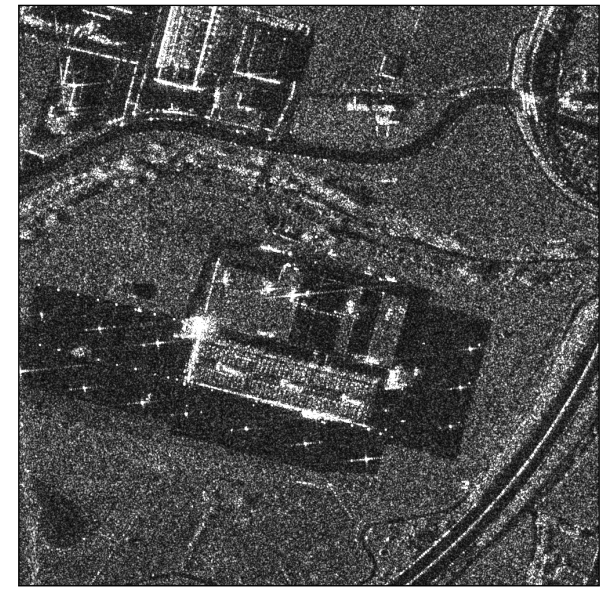

(a)

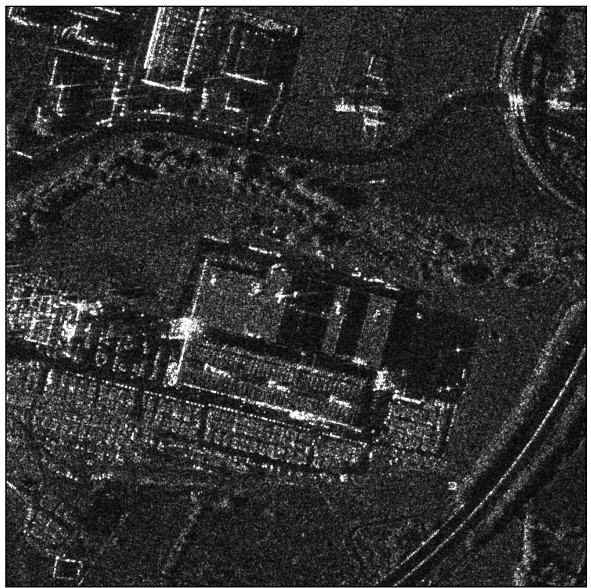

(b)

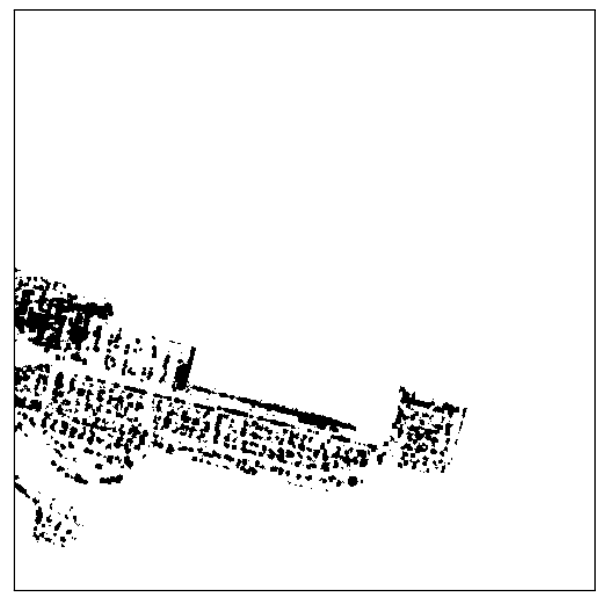

(c)

Fig. 12 Original pre- and post-event Spotlight acquisitions of L'Aquila test site: (a) April 5th, 2009; (b); September 12th, 2009; manually generated ground truth of change, (c).

The MS-ITCD and GMBR change features computed on the image pair of Fig. 12 are reported in Fig. 13 showing similar responses to structural and statistical changes, but different dynamic ranges.

The final change maps obtained by applying MS-ITCD, GMBR, FFL-ARS1, and FFL-ARS2 are shown in Fig.14. The two original images have been pre-processed 


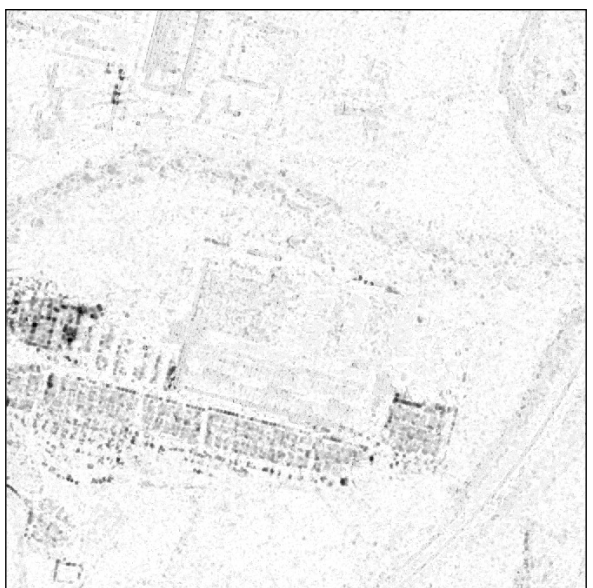

(a)

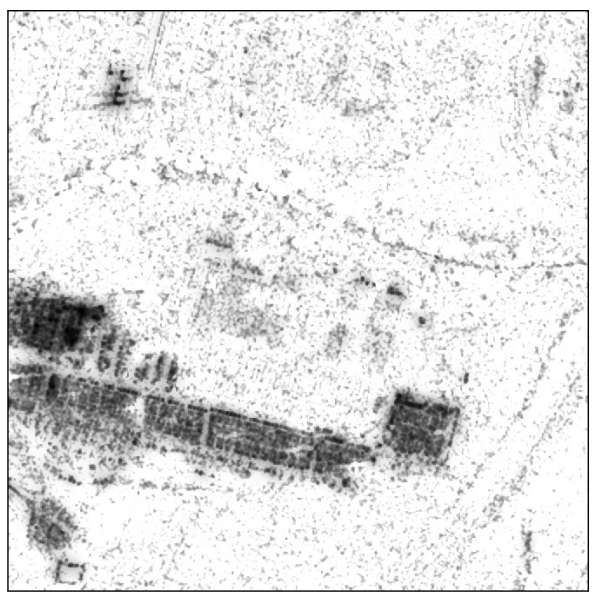

(b)

Fig. 13 Change features from the CSK image pair of Fig. 12: (a) MS-ITCD; (b) GMBR.

to equalise their histograms and the analysis has been focused on the regions with increased backscattering at the second date. All methods provide a clear description of the changed region with different results in terms of detection capability, false alarm rejection, and geometrical accuracy.

The analysis of the confusion matrices and the Cohen's kappa values provides an objective assessment of the characteristics of the three algorithms. The confusion matrices are the following:

$$
\begin{array}{rlrl}
C_{\mathrm{MS}-\mathrm{ITCD}} & =\left[\begin{array}{cc}
934874 & 17202 \\
12799 & 35125
\end{array}\right] & C_{\mathrm{FFL}-\mathrm{ARS} 1}=\left[\begin{array}{cc}
940501 & 7172 \\
30054 & 22273
\end{array}\right] \\
C_{\text {FFL-ARS2 }}=\left[\begin{array}{cc}
936092 & 11581 \\
18827 & 33500
\end{array}\right] & C_{\mathrm{GMBR}}=\left[\begin{array}{cc}
922963 & 24710 \\
15780 & 36547
\end{array}\right]
\end{array}
$$

which can be synthesised by the unique index $\kappa$ in Table 3 .

Table 3 Cohen's kappa values of the CD maps obtained from the CSK image pair.

\begin{tabular}{lr}
\hline Algorithm & Cohen's kappa \\
\hline MS-ITCD & 0.685 \\
GMBR & 0.622 \\
FFL-ARS1 & 0.527 \\
FFL-ARS2 & 0.672 \\
\hline
\end{tabular}



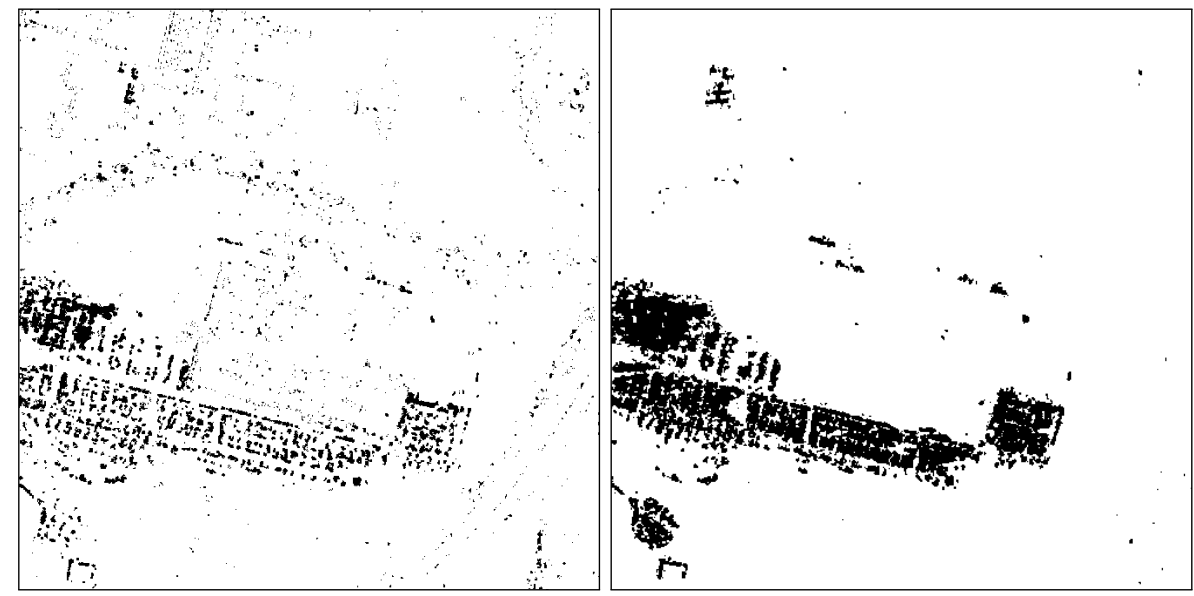

(a)

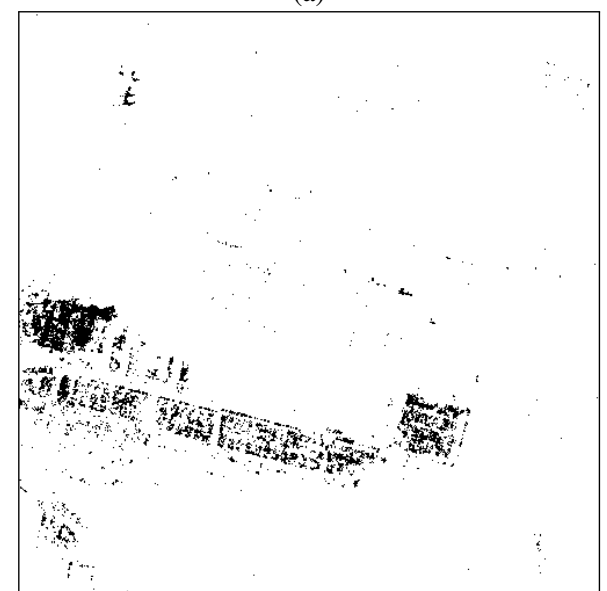

(c)

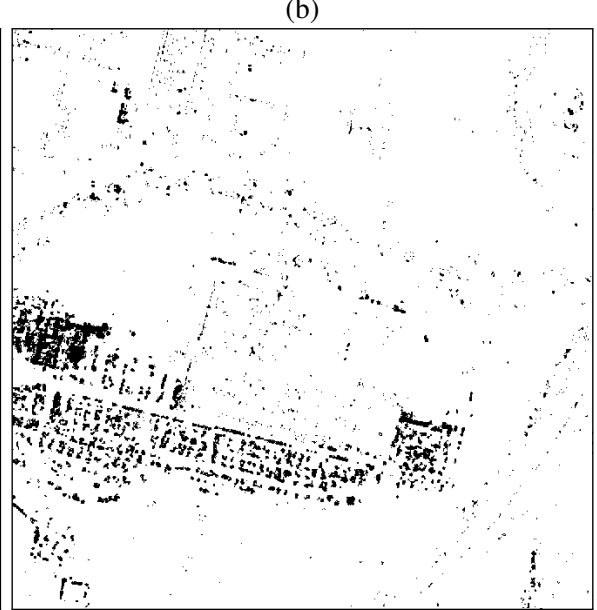

(d)

Fig. 14 Change maps obtained from the L'Aquila test site: (a) MS-ITCD; (b) GMBR; (c) FFLARS1; (d) FFL-ARS2.

The relatively low $\kappa$ values are due to inaccuracies of the ground truth which reports changes in the tent camp area only.

FFL-ARS2 and MS-ITCD show the highest $\kappa$ values, thanks to the MS-ITCD accurate detection of distributed scatterers, which provides a very good preservation of small spatial features in the changed regions. FFL-ARS1 suffers from severe missdetection due to its underlying log-ratio feature, while GMBR, although its change map appears clean and accurate at a first sight, is not capable of precisely locate the small distributed scatterers which characterise the change regions of the tested CSK image pair. 


\section{Concluding Remarks}

The capabilities of both single-scale and multiscale approaches of detecting changes from two-date SAR acquisitions have been investigated and experimentally assessed on 1-look and 4-look simulated images and on COSMO-Skymed Spotlight SAR data. It has been shown that when application-oriented prior information is not available for modeling different kinds of changes, multiscale approaches can be profitably applied to detect changes directly from the image radiometric properties at different dates. Among the tested algorithms, the single-scale MS-ITCD method has shown very accurate detection of distributed scatterers, the multiscale FFL-ARS algorithm, when driven by advanced change features such as MS-ITCD, has evidenced good shape preservation, while the multiscale GMBR algorithm has demonstrated the best tradeoff, for both simulated and true data, between speckle reduction and preservation of geometrical details.

\section{References}

1. Achim A, Kuruoglu EE, Zerubia J (2006) SAR image filtering based on the heavy-tailed Rayleigh model. IEEE Trans Image Process 15(9):2686-2693

2. Aiazzi B, Alparone L, Baronti S, Garzelli A, Nencini F (2008) Informationtheoretic multitemporal features for change analysis from SAR images. In: Image and Signal Processing for Remote Sensing XIV, vol 7109, p 71090S

3. Aiazzi B, Alparone L, Baronti S, Garzelli A, Zoppetti C (2011) A robust change detection feature for COSMO-SkyMed detected SAR images. In: Proc. MultiTemp 2011, International Workshop on the Analysis of Multi-temporal Remote Sensing Images, pp 125-128

4. Aiazzi B, Alparone L, Baronti S, Garzelli A, Zoppetti C (2013) Nonparametric change detection in multitemporal SAR images based on mean-shift clustering. IEEE Trans Geosci Remote Sens 51(4):2022-2031

5. Alparone L, Aiazzi B, Baronti S, Garzelli A (2006) An information-theoretic feature for multi-temporal analysis of SAR images. In: Proc. ESA-EUSC 2006: Image Information Mining for Security and Intelligence, ESA Workshop Proceedings Publication WPP-274, pp 67-76

6. Alparone L, Aiazzi B, Baronti S, Garzelli A, Nencini F (2007) Robust change analysis of SAR data through information-theoretic multitemporal features. In: Proc. IEEE Int. Geosci. Remote Sens. Symp., pp 3883-3886

7. Ban Y, Yousif OA (2012) Multitemporal spaceborne SAR data for urban change detection in China. IEEE J Sel Top Appl 5(4):1087-1094

8. Bazi Y, Bruzzone L, Melgani F (2005) An unsupervised approach based on the generalized Gaussian model to automatic change detection in multitemporal SAR images. IEEE Trans Geosci Remote Sens 43(4):874-887

9. Bezdek JC, Ehrlich R, Full W (1984) FCM: The fuzzy c-means clustering algorithm. Comput Geosci 10(2-3):191-203 
10. Bovolo F (2009) A multilevel parcel-based approach to change detection in very high resolution multitemporal images. IEEE Geosci Remote Sens Lett 6(1):33-37

11. Bovolo F, Bruzzone L (2005) A detail-preserving scale-driven approach to change detection in multitemporal SAR images. IEEE Trans Geosci Remote Sens 43(12):2963-2972

12. Bovolo F, Bruzzone L (2007) A theoretical framework for unsupervised change detection based on change vector analysis in the polar domain. IEEE Trans Geosci Remote Sens 45(1):218-236

13. Bovolo F, Marchesi S, Bruzzone L (2012) A framework for automatic and unsupervised detection of multiple changes in multitemporal images. IEEE Trans Geosci Remote Sens 50(6):2196-2212

14. Bovolo F, Marin C, Bruzzone L (2013) A hierarchical approach to change detection in very high resolution SAR images for surveillance applications. IEEE Trans Geosci Remote Sens 51(4):2042-2054

15. Brunner D, Lemoine G, Bruzzone L (2010) Earthquake damage assessment of buildings using VHR optical and SAR imagery. IEEE Trans Geosci Remote Sens 48(5):2403-2420

16. Bruzzone L, Bovolo F (2013) A novel framework for the design of changedetection systems for very-high-resolution remote sensing images. Proc IEEE 101(3):609-630

17. Bruzzone L, Cossu R (2003) An adaptive approach to reducing registration noise effects in unsupervised change detection. IEEE Trans Geosci Remote Sens 41(11):2455-2465

18. Bruzzone L, Fernández Prieto D (1999) A technique for the selection of kernelfunction parameters in RBF neural networks for classification of remote-sensing images. IEEE Trans Geosci Remote Sens 37(2):1179-1184

19. Bruzzone L, Fernández Prieto D (2000) Automatic analysis of the difference image for unsupervised change detection. IEEE Trans Geosci Remote Sens 38(3):1171-1182

20. Bruzzone L, Fernández Prieto D (2001) Unsupervised retraining of a maximum likelihood classifier for the analysis of multitemporal remote sensing images. IEEE Trans Geosci Remote Sens 39(2):456-460

21. Bruzzone L, Fernández Prieto D (2002) An adaptive semiparametric and context-based approach to unsupervised change detection in multitemporal remote-sensing images. IEEE Trans Image Process 11(4):452-466

22. Bruzzone L, Serpico SB (1997) An iterative technique for the detection of landcover transitions in multitemporal remote-sensing images. IEEE Trans Geosci Remote Sens 35(4):858-867

23. Bruzzone L, Fernández Prieto D, Serpico SB (1999) A neural-statistical approach to multitemporal and multisource remote-sensing image classification. IEEE Trans Geosci Remote Sens 37(3):1350-1359

24. Bruzzone L, Cossu R, Vernazza G (2004) Detection of land-cover transitions by combining multidate classifiers. Pattern Recogn Lett 25(13):1491-1500 
25. Carincotte C, Derrode S, Bourennane S (2006) Unsupervised change detection on sar images using fuzzy hidden markov chains. IEEE Trans Geosci Remote Sens 44(2):432-441

26. Celik T, Ma KK (2010) Unsupervised change detection for satellite images using dual-tree complex wavelet transform. IEEE Trans Geosci Remote Sens 48(3): 1199-1210

27. Celik T, Ma KK (2011) Multitemporal image change detection using undecimated discrete wavelet transform and active contours. IEEE Trans Geosci Remote Sens 49(2):706-716

28. Chen J, Chen X, Cui X, Chen J (2011) Change vector analysis in posterior probability space: A new method for land cover change detection. IEEE Geosci Remote Sens Lett 8(2):317-321

29. Chini M, Pulvirenti L, Pierdicca N (2012) Analysis and interpretation of the COSMO-SkyMed observations of the 2011 Japan tsunami. IEEE Geosci Remote Sens Lett 9(3):467-471

30. Cihlar J, Pultz TJ, Gray A (1992) Change detection with synthetic aperture radar. Int J Remote Sens 13(3):401-414

31. Cossu R, Chaudhuri S, Bruzzone L (2005) A spatial-contextual partially supervised classifier based on markov random fields. IEEE Geosci Remote Sens Lett pp 352-356

32. Cover TM, Thomas JA (2006) Elements of Information Theory, 2nd edn. Wiley, New York

33. Cristianini N, Shawe-Taylor J (2000) An introduction to support vector machines and other kernel-based learning methods. Cambridge University Press

34. Cui S, Datcu M (2012) Statistical wavelet subband modeling for multi-temporal SAR change detection. IEEE J Sel Top Appl 5(4):1095-1109

35. Dalla Mura M, Benediktsson JA, Bovolo F, Bruzzone L (2008) An unsupervised technique based on morphological filters for change detection in very high resolution images. IEEE Geosci Remote Sens Lett 5(3):433-437

36. Dekker RJ (1998) Speckle filtering in satellite SAR change detection imagery. Int J Remote Sens 19(6):1133-1146

37. Dekker RJ (2011) High-resolution radar damage assessment after the earthquake in Haiti on 12 january 2010. IEEE J Sel Top Appl 4(4):960-970

38. Demir B, Bovolo F, Bruzzone L (2012) Detection of land-cover transitions in multitemporal remote sensing images with active-learning-based compound classification. IEEE Trans Geosci Remote Sens 50(5):1930-1941

39. Demir B, Bovolo F, Bruzzone L (2013) Classification of time series of multispectral images with limited training data. IEEE Trans Image Process 22(8):3219-3233

40. Demir B, Bovolo F, Bruzzone L (2013) Updating land-cover maps by classification of image time series: A novel change-detection-driven transfer learning approach. IEEE Trans Geosci Remote Sens 51(1):300-312

41. Dippel S, Stahl M, Wiemker R, Blaffert T (2002) Multiscale contrast enhancement for radiographies: Laplacian pyramid versus fast wavelet transform. IEEE Trans Med Imag 21(4):343-353 
42. Duda RO, Hart PE, Stork DG (2012) Pattern classification. John Wiley \& Sons

43. Erten E, Reigber A, Ferro-Famil L, Hellwich O (2012) A new coherent similarity measure for temporal multichannel scene characterization. IEEE Trans Geosci Remote Sens 50(7):2839-2851

44. Falco N, Dalla Mura M, Bovolo F, Benediktsson JA, Bruzzone L (2013) Change detection in VHR images based on morphological attribute profiles. IEEE Geosci Remote Sens Lett 10(3):636-640

45. Garzelli A, Zoppetti C (2016) A segmentation-based approach to SAR change detection and mapping. In: Proc. SPIE 10004, Image and Signal Processing for Remote Sensing XXII, pp 1000,410-1000,410-10

46. Gueguen L, Soille P, Pesaresi M (2011) Change detection based on information measure. IEEE Trans Geosci Remote Sens 49(11):4503-4515

47. Hay GJ, Castilla G, Wulder MA, Ruiz JR (2005) An automated object-based approach for the multiscale image segmentation of forest scenes. Int J Appl Earth Obs 7(4):339-359

48. Inglada J, Mercier G (2007) A new statistical similarity measure for change detection in multitemporal SAR images and its extension to multiscale change analysis. IEEE Trans Geosci Remote Sens 45(5):1432-1445

49. Jeon B, Landgrebe DA (1992) Classification with spatio-temporal interpixel class dependency contexts. IEEE Trans Geosci Remote Sens 30(4):663-672

50. Klaric MN, Claywell BC, Scott GJ, Hudson NJ, Sjahputera O, Li Y, Barratt ST, Keller JM, Davis CH (2013) GeoCDX: An automated change detection and exploitation system for high-resolution satellite imagery. IEEE Trans Geosci Remote Sens 51(4):2067-2086

51. Kosko B (1992) Neural networks and fuzzy systems: a dynamical systems approach to machine intelligence. Prentice Hall, Upper Saddle River

52. Li S, Fang L, Yin H (2012) Multitemporal image change detection using a detailenhancing approach with nonsubsampled contourlet transform. IEEE Geosci Remote Sens Lett 9(5):836-840

53. Liu S, Bruzzone L, Bovolo F, Du P (2014) A novel sequential spectral change vector analysis for representing and detecting multiple changes in hyperspectral images. In: Proc. IEEE IGARSS' 14, pp 4656-4659

54. Liu S, Bruzzone L, Bovolo F, Du P (2015) Hierarchical unsupervised change detection in multitemporal hyperspectral images. IEEE Trans Geosci Remote Sens 53(1):244-260

55. Liu S, Bruzzone L, Bovolo F, Zanetti M, Du P (2015) Sequential spectral change vector analysis for iteratively discovering and detecting multiple changes in hyperspectral images. IEEE Trans Geosci Remote Sens 53(8):4363-4378

56. Marin C, Bovolo F, Bruzzone L (2015) Building change detection in multitemporal very high resolution SAR images. IEEE Trans Geosci Remote Sens 53(5):2664-2682

57. Mason DC, Speck R, Devereux B, Schumann GJP, Neal JC, Bates PD (2010) Flood detection in urban areas using TerraSAR-X. IEEE Trans Geosci Remote Sens 48(2):882-894 
58. Mercier G, Moser G, Serpico SB (2008) Conditional copulas for change detection in heterogeneous remote sensing images. IEEE Trans Geosci Remote Sens 46(5):1428-1441

59. Moser G, Angiati E, Serpico SB (2011) Multiscale unsupervised change detection on optical images by markov random fields and wavelets. IEEE Geosci Remote Sens Lett 8(4):725-729

60. Nielsen AA (2007) The regularized iteratively reweighted mad method for change detection in multi-and hyperspectral data. IEEE Trans Image Process 16(2):463-478

61. Oliver C, Quegan S (2004) Understanding synthetic aperture radar images. SciTech Publishing

62. Richards JA (2013) Remote sensing digital image analysis, 5th edn. SpringerVerlag, Berlin Heidelberg

63. Rignot EJ, van Zyl JJ (1993) Change detection techniques for ERS-1 SAR data. IEEE Trans Geosci Remote Sens 31(4):896-906

64. Schmitt A, Wessel B, Roth A (2014) An innovative curvelet-only-based approach for automated change detection in multi-temporal SAR imagery. MDPI Remote Sens 6(3):2435-2462

65. Serpico S, Bruzzone L (1999) Change detection. In: Chen CH (ed) Information processing for remote sensing, World Scientific, Ch.15

66. Singh A (1989) Digital change detection techniques using remotely-sensed data. Int J Remote Sens 10(6):989-1003

67. Solano Correa YT, Bovolo F, Bruzzone L (2014) Change detection in very high resolution multisensor optical images. In: Image and Signal Processing for Remote Sensing XX, SPIE Proc. 9244, pp 924,410-924,410

68. Solano Correa YT, Bovolo F, Bruzzone L (2015) VHR time-series generation by prediction and fusion of multi-sensor images. In: Proc. IEEE IGARSS'15, pp 3298-3301

69. Solano Correa YT, Bovolo F, Bruzzone L (2016) An approach to multiple change detection in multisensor VHR optical images based on iterative clustering. In: Proc. IEEE IGARSS'16, in press

70. Solberg AHS, Taxt T, Jain AK (1996) A markov random field model for classification of multisource satellite imagery. IEEE Trans Geosci Remote Sens 34(1):100-113

71. Vapnik V (2000) The nature of statistical learning theory, 2nd edn. SpringerVerlag, New York

72. Wang F (1990) Fuzzy supervised classification of remote sensing images. IEEE Trans Geosci Remote Sens 28(2):194-201

73. Zanetti M, Bovolo F, Bruzzone L (2015) Rayleigh-Rice mixture parameter estimation via EM algorithm for change detection in multispectral images. IEEE Trans Image Process 24(12):5004-5016 